\title{
IP Camera Video Surveillance using Raspberry Pi
}

\author{
Sneha Singh ${ }^{1}$, PradnyaAnap ${ }^{2}$, YogeshBhaigade ${ }^{3}$, Prof.J.P.Chavan ${ }^{4}$ \\ B.E Dept., of Computer Engg., Sinhgad Institute of Technology, Lonavala, Pune university, Maharashtra, India ${ }^{1,2,3}$ \\ Assistant professor, Computer dept, Sinhgad Institute of Technology, Lonavala, Pune university ${ }^{4}$
}

\begin{abstract}
Video Surveillance is important as far as security is concerned these days. Commercial spaces, schools and hospitals, warehouses and other challenging indoor and outdoor environments require high end cameras with PTZ. The current technologies require RFIDs which are costly and hence the security domain in all becomes expensive. This paper describes the use of low cost single -board computer Raspberry Pi which follows face detection algorithm written in Python as a default programming environment. This new technology is less expensive and in this paper it is used as a standalone platform for hosting image processing. The paper aims at developing a system which captures real time images and displays them on browser using TCP/IP. The algorithm for face detection is being implemented on raspberry pi which enables live video streaming along with detection of human faces.
\end{abstract}

Keywords: Raspberry Pi, Video surveillance, IP camera, embedded system

\section{INTRODUCTION}

Surveillance refers to observation and video surveillance thus refers to observation of real time video of a region from a remote area. Performance of the cameras thus becomes important. IP and PTZ cameras are used for deliverance of real time video remotely. However their usage is restricted when cost matter is concerned. Raspberry $\mathrm{Pi}$ is the least expensive technology whose usage is being proposed in this paper as an alternative for video surveillance. It is the cheapest and commercially available option when comparing with other alternatives. We present a new dimension in the security domain by utilising this mini board. This is not only cost effective but when the real time video quality is considered it delivers $120 \mathrm{x}$ cost effective per megapixel of resolution which is lower than other alternatives.

Most of the IP cameras require high bandwidth thus involving this as a major drawback. So indeed low cost video acquisition units are to be developed. Here we are proposing a system which connects the mini board with the web camera which captures the real time video and displays the detected number of human faces in the region to the browser. This real time video can be seen to the display monitor or on mobile devices in browser with the help localhost i.e. raspberry pi.Performance of the IP cameras are being judged with respect to its video resolution, power dissipation, bitrates and distortion, frame rates and network bandwidth. Compression ratio is also important when image quality is determined. Encoders play a major role with respect to these terms. This paper describes the use of cost effective BlackfinDsp and Raspberry Pi (ARM9) processor. The practical uses and performance tests reveal that IP cameras can easily deliver VGA or CIF size real time video clips directly to the browser. Thus application of these IP cameras in video surveillance may improve system quality and reliability and thereby lower the requirements of network bandwidth. Improving Coding efficiency is in direct relation to cost and computational complexity. So implementation of efficient encoders like H.264/AVC require larger memory space, more processor, efficient power dissipation etc.

This implies optimization should be developed in tandem with encoder, hardware and software. This paper describes a low profile web camera with an efficient ARM9 processor Raspberry Pi model. This camera can be configured remotely and captures real time video, encodes and delivers CIF and VGA pictures. The paper presented here is organised as follows. Section 2 briefs about mini board Raspberry Pi. In Section 3 we have stated about the camera implementation and configuration with the raspberry pi. Further Section 4 reveals Face Detection algorithm implemented in OpenCV. Haar Classifier Detection Algorithm is being described. The results are being displayed in the browser and conclusions are summarised in Section 5 and 6.

\section{OVERVIEW OF THE RASPBERRY PI \\ Raspberry Pi hardware}

The picture of Raspberry Pi is shown in Fig. 1. A normal web camera is used for live video capturing. The connections are also shown in the picture. This system proposed here uses Raspberry Pi Model B connected to a Logitech C300 universal serial bus (USB) webcam. Power supply required by the device is $3 \mathrm{~V} \mathrm{DC}$ which is being converted first from $5 \mathrm{~V}$ DC. External hard drive is required for data storage. Here 16 GB Secure Digital High Capacity transcend card is used. Internet connection is being provided via CAT6 Ethernet cable. The camera module is being attached to the stepper motor which is being controlled through the device. This is done to ensure that camera captures images in all directions. Raspberry Pi model is an ARM 9 processor with description and further specifications are shown in the table below.

\section{Raspberry Pi Software}

During initial setup Raspberry Pi was configured as a miniature desktop with USB configured camera module and an external monitor or mobile for viewing the 
captured video. Browser is used for viewing of live video stream. The external card is loaded with Raspbian OS. The Dynamic host Protocol is obtained for Raspberry Pi i.e IP addressed is fetched. After obtaining this the system can be configured and controlled remotely. The live video can be viewed by dialling-in the IP address into a web browser.

Cost of the whole system is $\$ 70$. Cost of Raspberry Pi is less as compared to other alternatives. We can calculate the cost-per-mega-pixel and conclude that image resolution on remote screen by raspberry pi is $960 \times 720$ (0.7 megapixels) and cost-per-megapixel image is as approximately is $\$ 125$.

\section{IMPLEMENTATION}

The project components which include raspberry pi, webcamera, monitor/mobile for display are shown in figure.

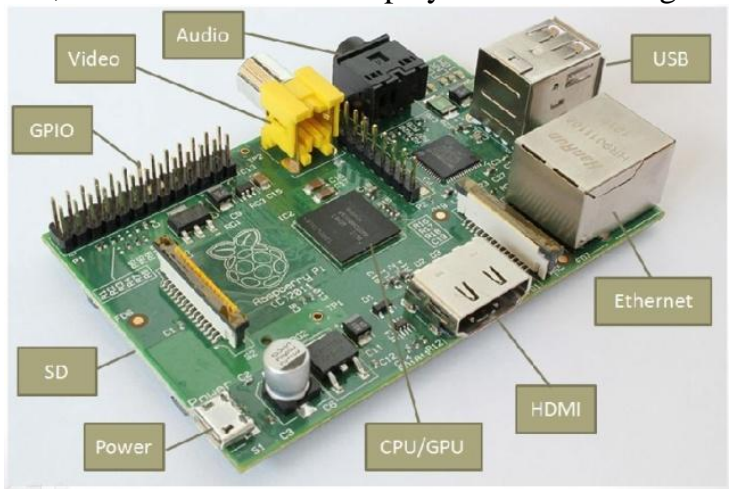

Fig. 1.Raspberry pi

As shown in Fig. 1, there are number of different ports available on raspberry pi such as video, audio as output ports, USB port for external device connection like camera, Ethernet port for net connection (i.e.Wifi module), HDMI port which is used for both audio and video(works with modern TVs and DVI monitors), power connection port requires $5 \mathrm{~V}$ power supply.

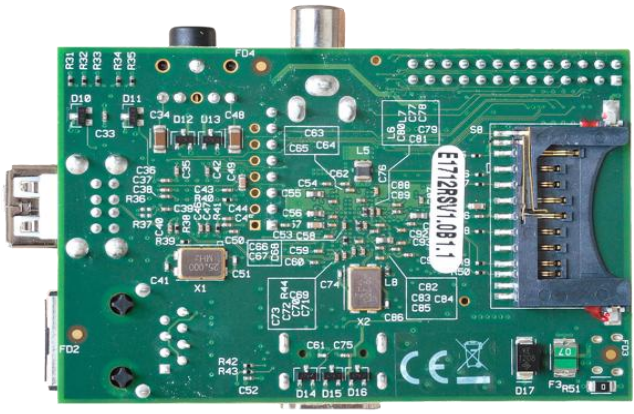

Fig. 2.Back side of raspberry

As shown in Fig. 2, SD card slot for storage purpose which supports up to $16 \mathrm{~GB}$.

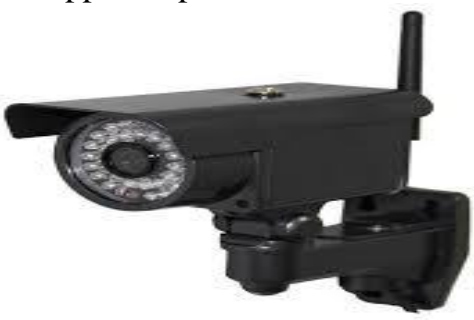

Fig. 3(a).IP Camera

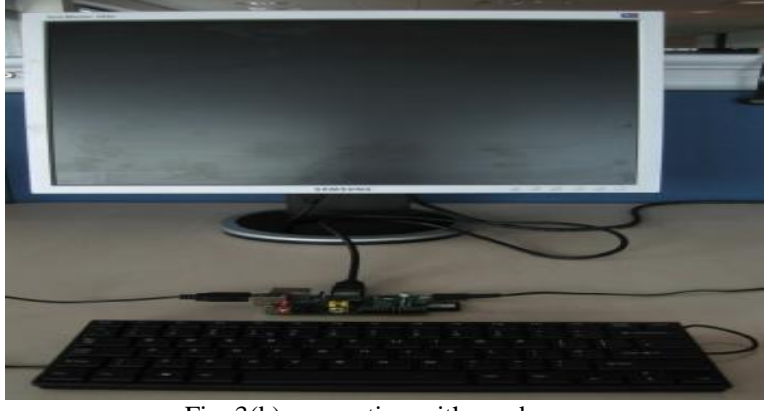

Fig. 3(b).connection with raspberry

Fig. 3(a) shows IP camera which works on IP and Fig. 3(b) shows the connection of raspberry pi, camera and PC.

\section{IV.}

\section{FACE DETECTION ALGORITHM}

The Haar classifier algorithm proposed by Viola and Jones is applied here in OpenCv. Haar like features are measured which are determined by contrasting the pixel variation in nearby pixel groups. The algorithm has mainly 4 stages:

1. Haar Features Selection

2. Creating Integral Image

3. Adaboost Training algorithm

4. Cascaded Classifiers

Haar features are being characterized by dividing the human face into different regions. The facial region is divided by considering the size and brightness variation and calculated as

Value $=\Sigma($ pixels in black area $)-\Sigma($ pixels in white area $)$
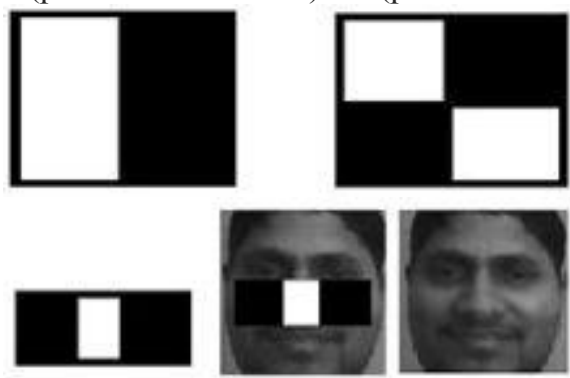

Also the integral image at a pixel at location $(x, y)$ is calculated which adds on to the efficiency of the algorithm. Next Adaboost algorithm is used which builds a strong classifier with multiple weak classifiers.

$$
h(\mathbf{x})=\operatorname{sign}\left(\sum_{j=1}^{M} \alpha_{j} h_{j}(\mathbf{x})\right)
$$

The different regions are being separated as facial as well as non-facial in each window. Further cascading is done with each window to improvise face detection. The following steps take place-

The following framework for cascade training is as follows:

- User selects values for $\mathrm{f}$, the maximum acceptable false positive rate per layer and $d$, the minimum acceptable detection rate per layer.

- User selects target overall false positive rate Ftarget.

- $\quad \mathrm{P}=$ set of positive examples

- $\quad \mathrm{N}=$ set of negative examples 
- $\quad \mathrm{F}(0)=1.0 ; \mathrm{D}(0)=1.0 ; \mathrm{i}=0$

$r$, the false positive rate for an entire cascade is:

$$
F=\prod_{i=1}^{K} f_{i} .
$$

Similarly, the detection rate is:

$$
D=\prod_{i=1}^{K} d_{i} .
$$

Thus to achieve a high detection the classifier must cascade and reach detection rate approximately $90 \%$.Hence the image is being detected and identified.

\section{FUTURE SCOPE}

The system described in the system used Raspberry Pi as a main device. However the drawback of the system can be realised as the system utilises Ethernet connection rather than wireless. Despite this drawback, the system can be used by remote user from any workstation within the network. Most of the laboratories use computers and switching to other alternative systems like Raspberry Pi would not be expensive. Automatic Toll Booth system, attendance monitoring system, and many other areas my use this device. Though other options like iPhone based systems are good ones but the device's extraordinary low cost makes it accessible for easy utilization. A robust face detection algorithm is difficult to design considering lot of tests and processes analysis.

\section{CONCLUSION}

The performance of the system can be enhanced by considering certain conditions. This may include mounting of light sources to improve lighting. Another improvement to the system can be done by creating automated test generation and analysis which eliminates need of operator. The application can be extended to additional GSM module and for extension of detection capabilities to various patterns not restricted to faces.

\section{ACKNOWLEDGEMENT}

This paper is an efficient contribution of its authors to the field of surveillance system. The work done here is expected to bring a change in the current trend of the system by switching to this method.

\section{REFERENCES}

[1] Priyanga .M, Raja ramanan .V "Unmanned Aerial Vehicle for VideoSurveillance Using Raspberry Pi" International Journal of Innovative Research in Science, Engineering and TechnologyVolume 3, Special Issue 3, March 2014.

[2] D.Jeevanand, K.Keerthivasan,J.MohamedRilwan,P.Murugan "Real Time Embedded Network Video Capture And SMS Alerting system" International Journal of Communication and Computer Technologies, 2014.

[3] Minh-Son Dao*, Riccardo Mattivi, Francesco G.B. De Natale, Keita Masui*, Noboru Babaguchi "Abandoned Object's Owner Detection: A Case Study of Hybrid Mobile-fixed Video Surveillance System" 978-0-7695-4797-8/12 \$26.00 (C) 2012 IEEEDOI 10.1109/AVSS.2012.4.

[4] Chia-Feng Lin, Muh-ChyiLeu, Shyan-Ming Yuan, Ching-Tsorng Tsai, "A framework for scalable cloud video recorder system in surveillance environment" 978-0-7695-4843-2/12 \$26.00 (C) 2012 IEEE DOI 10.1109/UIC-ATC.2012.72.

[5] PetreAnghelescu, *IonutSerbanescu, SilviuIonita "Surveillance System using IP Camera and Face-Detection Algorithm" 978-14673-4937-6/13/\$31.00 @2013IEEE.

[6] Muhammad JunaidMuzammil, Athar Mahboob, "Design of Multithreaded Real Time Embedded Video Acquisition System from IP Cameras" 978-1-4673-5885-9/13/\$31.00 @ 2013 IEEE.

[7] SayantaniSaha, SarmisthaNeogy, "A Case Study on smart Surveillance ApplicationSystem using WSN and IP webcam" 9781-4799-3880-3/14/\$31.00 (C) 2014 IEEE.

[8] FotisAndritsopoulos, SerafeimPapastefanos, George Georgakarakos, Gregory Doumenis "RELIABLE MULTICAST H.264 VIDEO $\quad$ STREAMING FOR SURVEILLANCEAPPLICATIONS" 1-4244-11440/07/\$25.0002007 IEEE.

[9] Ming-Jiang Yang, Jo Yew Tham, Dajun Wu, Kwong Huang Goh "Cost Effective IP Camera for Video Surveillance" 978-1-42442800-7/09/\$25.00 @2009 IEEE.

[10] Rhys Hill, Christopher Madden, Anton van den Hengel, Henry Detmold, Anthony Dick "Measuring Latency for Video Surveillance Systems" 978-0-7695-3866-2/09 \$26.00 @ 2009 IEEE DOI 10.1109/DICT A.2009.23 